\title{
A STUDY OF THE REACTION $\pi^{-} \mathrm{p} \rightarrow \pi^{-} \pi^{+} \mathrm{n}$ AT $16 \mathrm{GeV} / \mathrm{c}^{*}$
}

J. Ballam, G. B. Chadwick, Z.G.T. Guiragossián, W. B. Johnson, D.W.G.S. Leith and K. Moriyasu

Stanford Linear Accelerator Center Stanford University, Stanford, California 94305

\begin{abstract}
We present an analysis of the reaction $\pi^{-} \mathrm{p} \rightarrow \pi^{-} \pi^{+} \mathrm{n}$ at $16 \mathrm{GeV} / \mathrm{c}$. The total cross section for this channel was measured to be $0.40 \pm 0.08 \mathrm{mb}$ and production cross sections of $52 \pm 13 \mu \mathrm{b}$ and $38 \pm 9 \mu \mathrm{b}$ were determined for the $\rho^{\circ}$ and $f^{\circ}$ resonances, respectively. A comparison with the one-pion-exchange model of Wolf shows good agreement with our data.
\end{abstract}

(Submitted to Phys. Letters B.)

\footnotetext{
Work supported by the U. S. Atomic Energy Commission.
} 
In this letter we report a study of the reaction

$$
\pi^{-} p \rightarrow \pi^{-} \pi^{+} n
$$

at $16 \mathrm{GeV} / \mathrm{c}$. We have measured the total cross section for this reaction and the production cross sections for the $\rho^{0}$ and $f^{0}$ resonances in the $\pi^{-} \pi^{+}$system. We compare our data with the one-pion (OPE) exchange model of Wolf ${ }^{1}$ since it has had considerable success in describing a large class of one-pion exchange reactions over a wide range of energies. The predicted cross sections as well as many of the detailed features of the reaction agree well with our data.

The data were obtained from a 60,000-picture exposure of the Brookhaven 80 -inch hydrogen bubble chamber to a $16 \mathrm{GeV} / \mathrm{c} \pi^{-}$beam. All two-prong events were fitted using the TVGP-SQUAW system of programs and checked for consistency with the ionization values calculated by the fit. In order to obtain a clean sample of data for reaction (1) we retained only those events which had no alternative elastic fit but which could have one or more inelastic fits. A cut was imposed on the missing mass of the remaining data to remove events of the type $\pi^{-} \mathrm{p} \rightarrow \pi^{-} \pi^{+} \mathrm{n}\left(\pi^{0}\right)$ which had been misclassified as reaction (1). The most numerous of the competing inelastic fits were the final states $\mathrm{p} \pi^{-} \pi^{\circ}, \mathrm{K}^{+} \mathrm{K}^{-} \mathrm{n}$ and $\mathrm{pp}$. Essentially all of the $\mathrm{p} \pi^{-} \pi^{\circ}$ and more than half of the $\mathrm{K}^{+} \mathrm{K}^{-} \mathrm{n}$ and $\mathrm{pp}$. fits were eliminated by the missing mass cut and by a confidence level cut. The amount of remaining contamination, mostly from the $\mathrm{K}^{+} \mathrm{K}^{-} \mathrm{n}$ and $\mathrm{p} \overline{\mathrm{p}} \mathrm{n}$ fits, was determined from measurements at lower energies by assuming that the cross sections decrease at least linearly with the beam momentum. An upper limit of $10 \%$ was estimated for contamination from all inelastic fits and this value was used for correcting cross sections. The final sample of data contained 573 events fitting reaction (1) after all cuts were made. 
The total cross section for reaction (1) is $0.40 \pm 0.08 \mathrm{mb}$. This was determined from our measured total two-prong topological cross section and also by fitting the four-momentum transfer distribution of the elastic scattering events normalized in the forward direction to the data of Foley et al. ${ }^{2}$ Both techniques were used to assure a consistent result. The OPE model predicts a total cross section of $0.41 \mathrm{mb}$ at $16 \mathrm{GeV} / \mathrm{c}$ in good agreement with our value. Recently, Honecker et al. $^{3}$ also measured the total cross section of reaction (1) and obtained a value of $0.27 \pm 0.10 \mathrm{mb}$ which is somewhat lower but still consistent with our result.

Figure 1 shows the invariant mass spectra for the $\pi^{+} \pi^{-}, \pi^{+} \mathrm{n}$ and $\pi^{-} \mathrm{n}$ systems. The $\rho^{\circ}$ and $f^{\circ}$ mesons are clearly produced and there is some evidence for the $\mathrm{g}^{\mathrm{o}}$ resonance at a mass of $\sim 1700 \mathrm{MeV} / \mathrm{c}^{2}$. The $\pi^{+} \mathrm{n}$ spectrum is dominated by a large enhancement at low $\pi^{+} \mathrm{n}$ masses while no corresponding effect appears in the $\pi^{-} \mathrm{n}$ mass distribution. It has been suggested ${ }^{4}$ that part of the $\pi^{+} n$ peak could be due to contributions from the known nucleon isobars; however, none of the existing data show any clear separation of resonance peaks in the $\pi^{+} n$ mass spectrum.

The solid curves in Fig. 1 are the OPE predictions which agree well with the absolute cross section and the general features of the $\pi^{-} \pi^{+}, \pi^{+} n$ and $\pi^{-} n$ mass distributions. In particular, the concentration of events at low $\pi^{+} n$ masses is explained by the OPE model as primarily due to kinematical reflections from the dipion spectrum. We can understand this, at least qualitatively, from the observation that there is a strong correlation of high dipion masses with low $\pi^{+} n$ masses in the data. The high-energy pion-pion interaction is essentially diffractive which causes the $\pi^{+}$to be produced predominantly backward with respect to the beam or at low momentum relative to the recoil neutron. This results in more events with low values of the $\pi^{+} n$ invariant mass. Conversely, the forward produced $\pi^{-}$leads to high $\pi^{-} \mathrm{n}$ mass values and a distribution which is more like phase-space. 
An alternative explanation of the low mass $\pi^{+} n$ peak has been suggested by Walker et al. ${ }^{4}$ on the basis of their study of reaction (1) at 7 and $25 \mathrm{GeV} / \mathrm{c}$. They conclude that a diffraction scattering process is present in addition to onepion exchange in reaction (1) and that the $\pi^{+} n$ enhancement is the result of diffraction dissociation of the target nucleon. We suggest that this picture may be simply an interpretation of one feature of reaction (1) that is still completely consistent with the OPE model. As mentioned above, the high energy $\pi-\pi$ interaction is essentially diffractive so that the $\pi-\pi$ scattering amplitude above the resonance region can be approximated by Pomeron exchange. The complete OPE diagram in Fig. 2a thus contains a contribution for high dipion masses that can be associated with the usual diffraction dissociation diagram shown in Fig. $2 \mathrm{~b}$.

The highly peripheral nature of reaction (1) is evident in Fig. 3 which shows the distribution of the four-momentum transfer $t$ to the recoil neutron for the entire reaction (a) and for the $\rho^{0}$ and $f^{0}$ resonances, ${ }^{5}$ (b) and (c), respectively. The absolute OPE predictions, shown as solid curves in Fig. 3, agree well with the data for the entire reaction and are consistent, within the limited statistics, with the t-distributions of the $\rho^{\circ}$ and $\mathrm{f}^{\mathrm{O}}$.

Some shrinking of the width of the forward peak at high energy is predicted by Regge-pole theory if the reaction is dominated by the exchange of a single trajectory. If we describe the differential cross section by the function $\exp [-\lambda|t|]$ in the region of small $|t|$, we estimate a slope $\lambda$ of approximately $11-15(\mathrm{GeV} / \mathrm{c})^{-2}$ for the $\rho^{\circ}$ and $f^{\circ}$. This is compatible with the values obtained at other energies which seems to indicate that there is little or no shrinking of the forward peak with increasing energy. However, we note that simple Reggepole theory which predicts $\lambda \sim \ln s$ requires that $\lambda$ change only from 8 to 11 $(\mathrm{GeV} / \mathrm{c})^{-2}$ as the beam energy increases from 8 to $16 \mathrm{GeV} / \mathrm{c}$ so that the accuracy of existing data is not sufficient to decide the question of shrinkage. 
The production cross sections for the $\rho^{0}$ and $\mathrm{f}^{\mathrm{o}}$ mesons were determined by fitting the $\pi^{-} \pi^{+}$mass spectrum with Breit-Wigner resonances plus a non-interfering background. A simple polynomial and a statistical phase-space weighted ${ }^{6}$ by a function $\exp [-\mathrm{a}|\mathrm{t}| \mathrm{]}$ were both used to describe the background. The values of the fitted cross sections were found to be insensitive to any differences in the two background forms used. The production cross sections obtained were $\sigma\left(\rho^{0}\right)=52 \pm 13 \mu \mathrm{b}$ and $\sigma\left(f^{0}\right)=38 \pm 9 \mu \mathrm{b}$. The $\mathrm{g}^{0}$ was not fitted due to the low statistics and we give only an upper limit of $25 \pm 6 \mu \mathrm{b}$ for the events in the $\pi^{+} \pi^{-}$ mass region $1.6-1.9 \mathrm{GeV} / \mathrm{c}^{2}$. The absolute OPE predictions for the resonance cross sections are $\sigma\left(\rho^{\circ}\right)=51 \mu \mathrm{b}$ and $\sigma\left(\mathrm{f}^{\circ}\right)=43 \mu \mathrm{b}$.

We compare our cross sections with those of previous experiments in Fig. 4 which shows a compilation ${ }^{7}$ of the $\pi^{-} p \rightarrow \pi^{-} \pi^{+} n, \pi^{-} p \rightarrow \rho^{0} n$ and $\pi^{-} p \rightarrow f^{0} n$ cross sections as a function of the laboratory momentum $\mathrm{p}_{\mathrm{lab}}$ of the incident pion. Except for a single point at $4 \mathrm{GeV} / \mathrm{c}$, all of the cross section data are compatible with a simple momentum dependence of the form ${ }^{8} \sigma \sim\left(p_{1 a b}\right)^{-n}$ where $n$ is equal to 1.3 for the $\pi^{-} \pi^{+} \mathrm{n}$ final state and 2 for the $\rho^{0}$ and $\mathrm{f}^{\mathrm{O}}$ mesons. The initial rise and turnover of the $f^{0}$ cross section is characteristic of a steeply falling matrix element cut off by phase-space limitations near threshold. The solid curves in Fig. 4 are the absolute OPE cross section predictions. Both the $\rho^{0}$ and $f^{0}$ curves reproduce the energy dependence of the data very well from nearly $2 \mathrm{GeV} / \mathrm{c}$ up to $16 \mathrm{GeV} / \mathrm{c}$. The good agreement is notable since the entire energy-dependence of the OPE model comes from the flux factor which has the form $\sim\left(\mathrm{p}_{1 \mathrm{ab}}\right)^{-2}$.

The OPE prediction for the entire reaction (1) also agrees reasonably well with the data, particularly at high energy. The much slower decrease with energy results from the integration over all dipion masses which introduces an extra 
factor $p_{\text {lab }}$ through the integration limits. The discrepancy at low energies indicates that processes other than one-pion exchange, such as formation of s-channel resonances, 9 are contributing to the reaction.

\section{Acknowledgements}

We wish to thank Dr. G. Wolf and Dr. F. Gilman for several helpful discussions. 


\section{REFERENCES}

1. G. Wolf, Phys. Rev. 182, 1538 (1969).

2. K. J. Foley et al., Phys. Rev. Letters 10, 376 (1963) .

3. R. Honecker et al., (Aachen-Berlin-Bonn-CERN-Cracow-HeidelbergWarsaw Collaboration), Nucl. Phys. B13, 571 (1969).

4. W. D. Walker et al., Phys. Rev. Letters 20, 133 (1968).

5. The mass regions used were $(0.6-0.9) \mathrm{GeV} / \mathrm{c}^{2}$ for the $\rho^{\circ}$ and $(1.1-1.4) \mathrm{GeV} / \mathrm{c}^{2}$ for the $\mathrm{f}^{\mathrm{o}}$.

6. The value used for a was $4.4(\mathrm{GeV} / \mathrm{c})^{-2}$, the average slope of the fourmomentum transfer distribution for dipion masses greater than $1.3 \mathrm{GeV} / \mathrm{c}^{2}$.

7. J. Alitti et al., Nuovo Cimento $29,515(1963)[1.59 \mathrm{GeV} / \mathrm{c}]$;

L. Jacobs, Report No. UCRL-16877 (unpublished) $[2.05-3.22 \mathrm{GeV} / \mathrm{c}]$;

B. G. Reynolds et al. , Phys. Rev. 184, 1424 (1969) $[2.26 \mathrm{GeV} / \mathrm{c}]$;

J. P. Baton et al. , Nuovo Cimento $\underline{35}, 713$ (1965) $[2.75 \mathrm{GeV} / \mathrm{c}]$; Aachen-Birmingham-Bonn-Hamburg-London-Munich Collaboration, Nuovo Cimento 31,279 (1964) $[4 \mathrm{GeV} / \mathrm{c}]$;

J. A. Poirer et al., Phys. Rev. 163, 1462 (1967) $[8 \mathrm{GeV} / \mathrm{c}]$;

P. Fleury et al., Proc. of the 1962 Int'1. Conf. on High-Energy Physics at CERN, p. $597[10 \mathrm{GeV} / \mathrm{c}]$;

C. Caso et al., Nuovo Cimento 42A, 755 (1969) $[11 \mathrm{GeV} / \mathrm{c}]$.

8. D. R. O. Morrison, Phys. Letters 22, 528 (1966).

9. B. G. Levi, private communication. 


\section{FIGURE CAPTIONS}

1. Mass spectra for the (a) $\pi^{-} \pi^{+}$; (b) $n \pi^{+}$; (c) $n \pi^{-}$systems. The solid curves are the absolute OPE-model predictions.

2. (a) The OPE diagram for the reaction $\pi^{-} \mathrm{p} \rightarrow \pi^{-} \pi^{+} \mathrm{n}$.

(b) Approximation of the OPE diagram for high-energy $\pi^{+} \pi^{-}$scattering by exchange of a Pomeron.

3. Differential cross sections $d \sigma / d|t|$ for the final states (a) $\pi^{-} \pi^{+} n$; (b) $\rho^{0} n$; (c) $\mathrm{f}^{\mathrm{o}} \mathrm{n}$. The solid curves are the absolute cross sections from the OPE model.

4. Compilation of the total cross section as a function of laboratory momentum, $\mathrm{p}_{\mathrm{lab}}$, for the reactions $\pi^{-} \mathrm{p} \rightarrow \pi^{-} \pi^{+} \mathrm{n}, \pi^{-} \mathrm{p} \rightarrow \rho^{\circ} \mathrm{n}$ and $\pi^{-} \mathrm{p} \rightarrow \mathrm{f}^{\mathrm{o}} \mathrm{n}$. The solid curves are the absolute OPE predictions. The dashed line drawn through the $\pi^{-} \mathrm{p} \rightarrow \pi^{-} \pi^{+} \mathrm{n}$ data points has a slope of approximately 1.3 . 

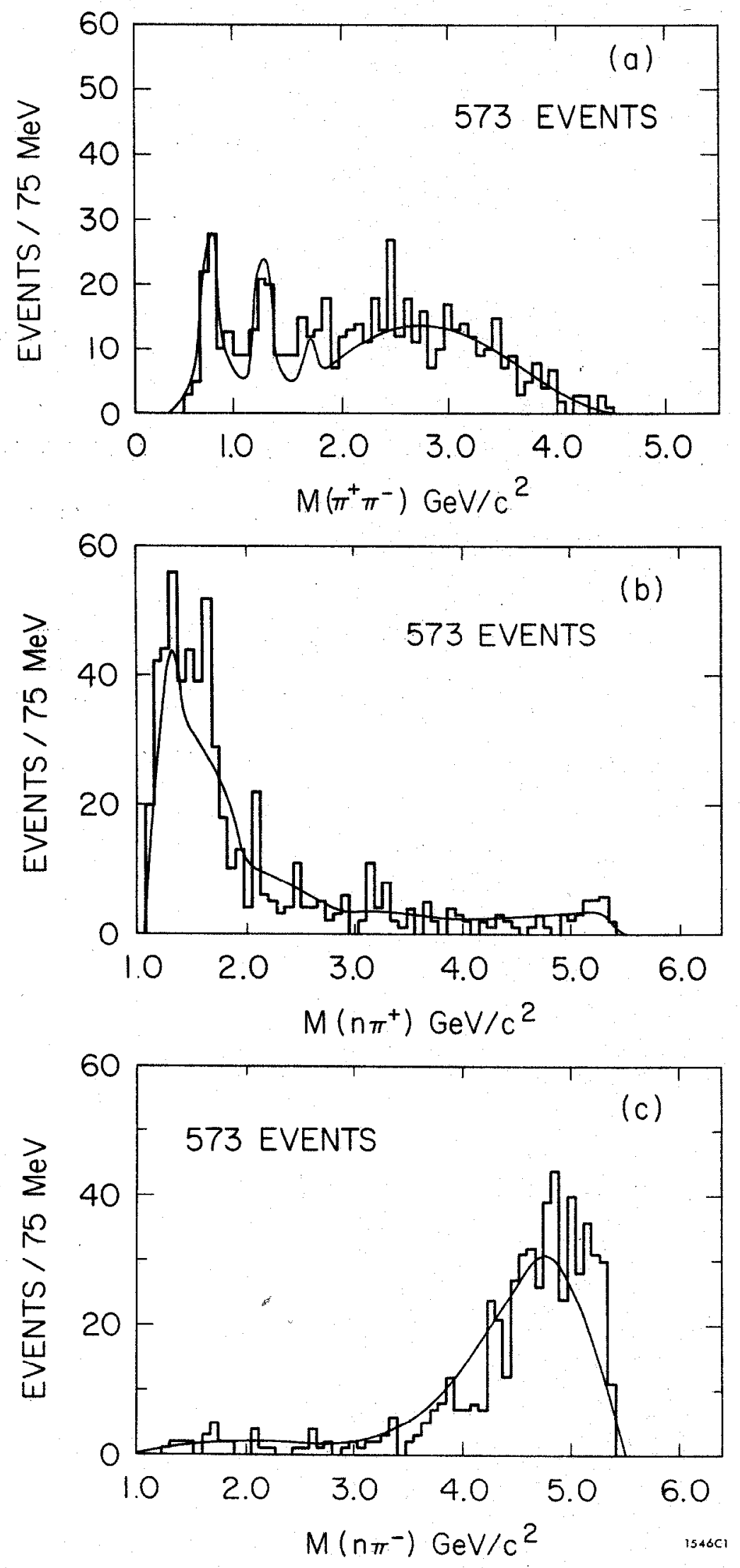

Fig. 1 
(a)

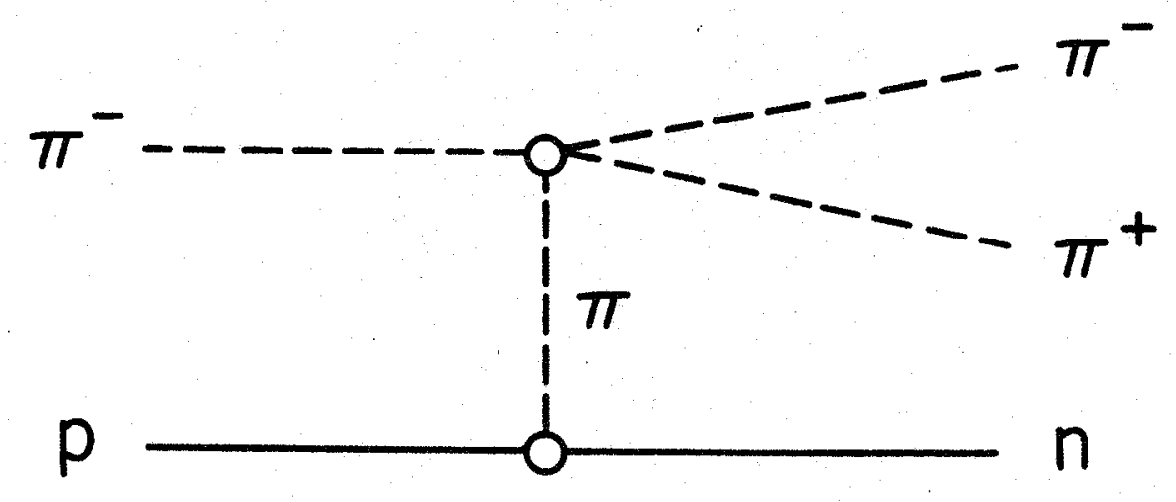

(b)

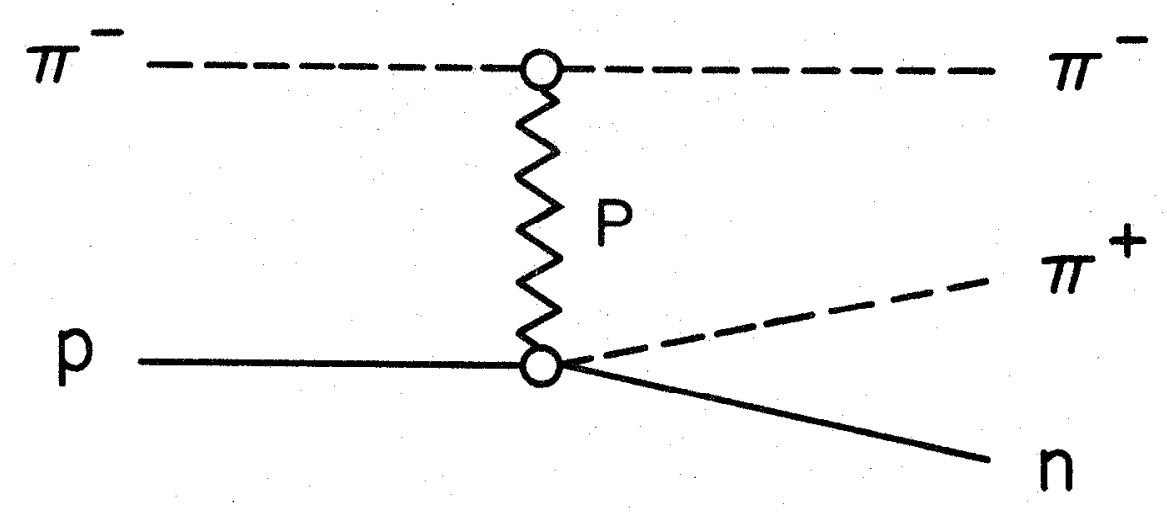

1546A2

Fig. 2 

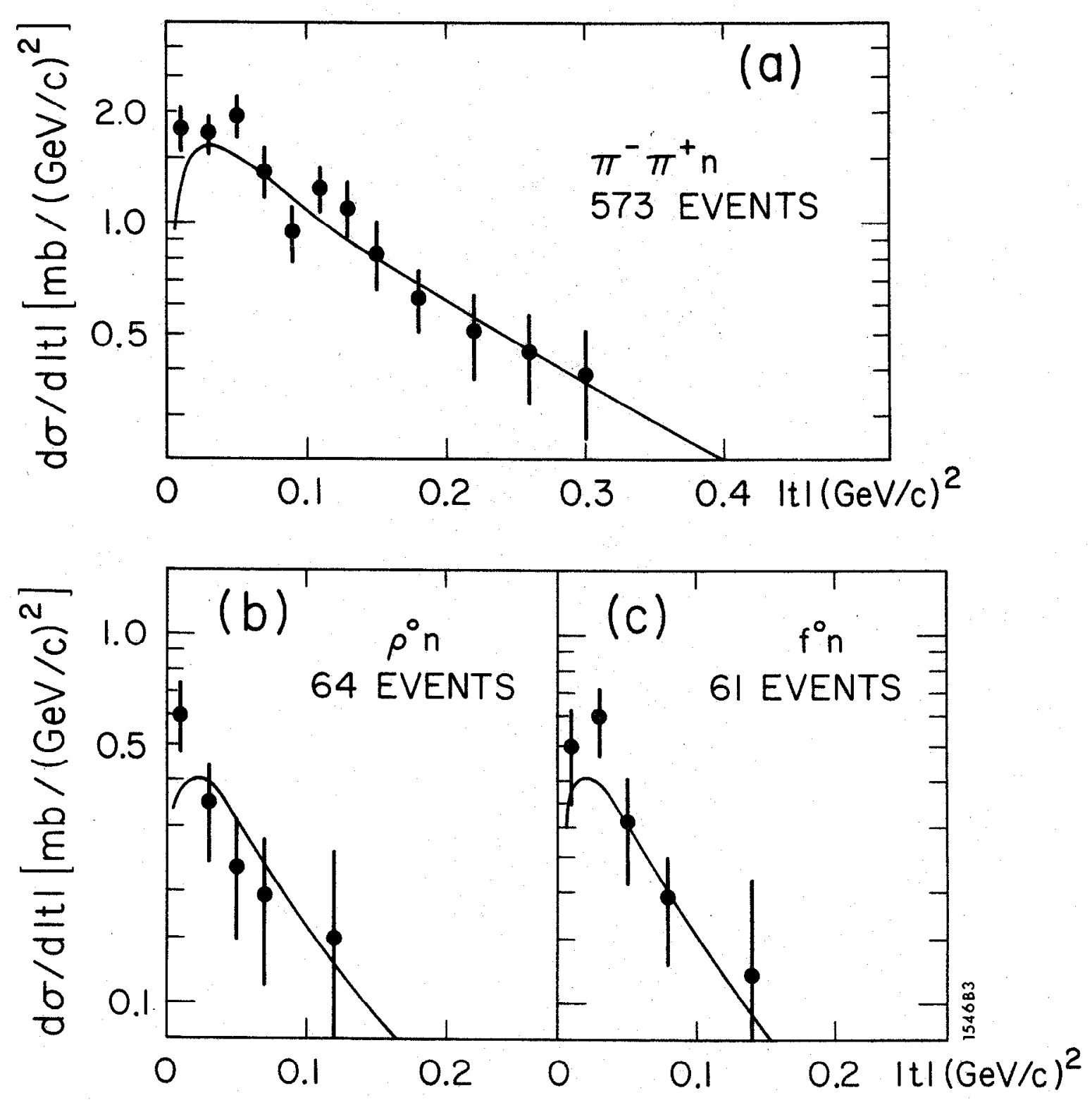

Fig. 3 


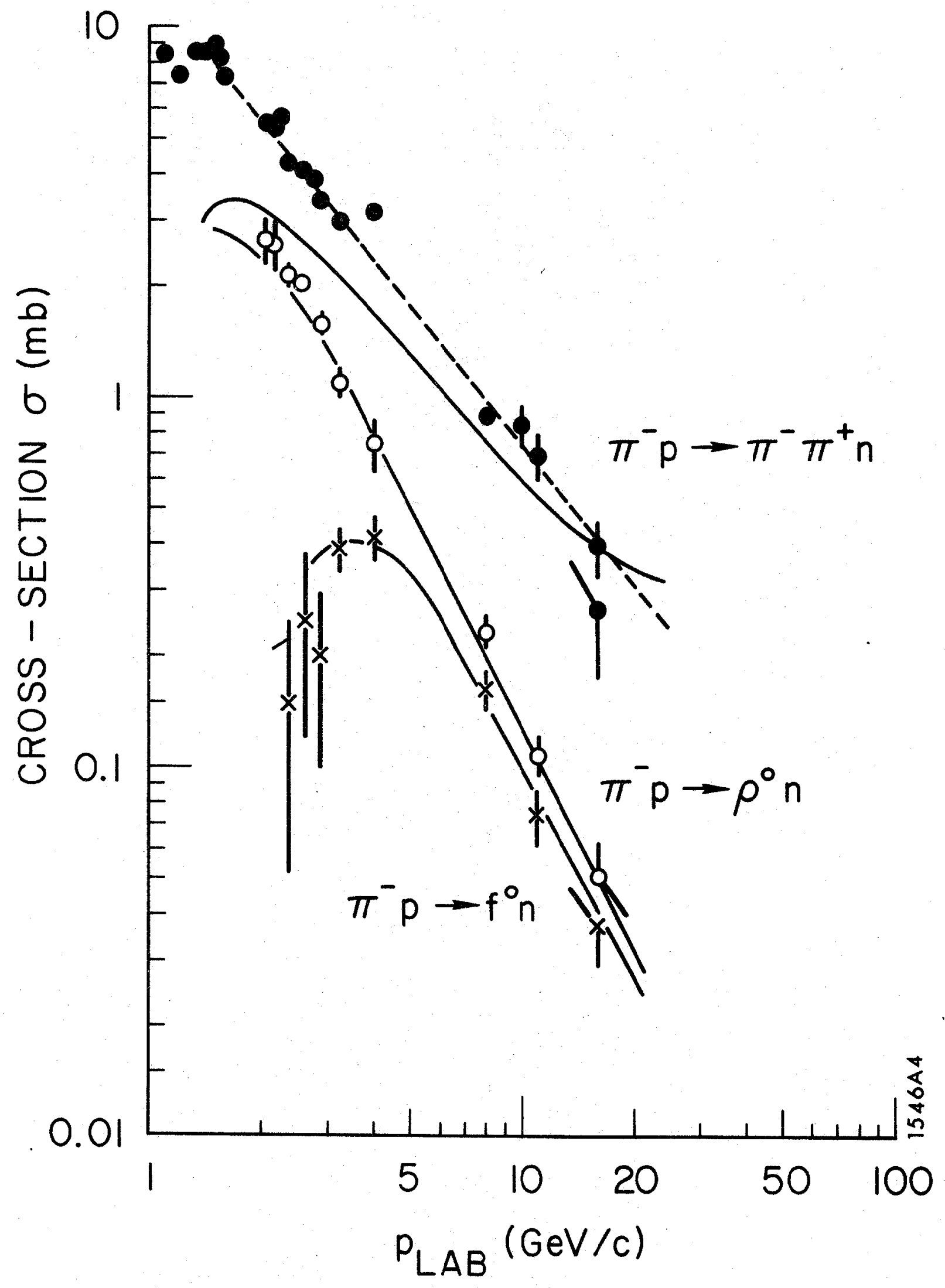

Fig. 4 